\title{
Tumour-Derived Reg3A Educates Dendritic Cells to Promote Pancreatic Cancer Progression
}

\author{
Jie Guo ${ }^{1,2,3}$, Mengfan Liao ${ }^{1,2,3}$, Xianmin $\mathrm{Hu}^{1,2,3}$, and Jun Wang ${ }^{1,2, *}$
}

\begin{abstract}
${ }^{1}$ Hubei Province Key Laboratory of Occupational Hazard Identification and Control, Wuhan University of Science and Technology, Wuhan 430065, China, ${ }^{2}$ New Medicine Innovation and Development Institute, Department of Pharmacy, College of Medicine, Wuhan University of Science and Technology, Wuhan 430065, China, ${ }^{3}$ These authors contributed equally to this work.

*Correspondence: wangjun@wust.edu.cn https://doi.org/10.14348/molcells.2021.0145

www.molcells.org
\end{abstract}

\begin{abstract}
As a pancreatic inflammatory marker, regenerating isletderived protein $3 \mathrm{~A}(\operatorname{Reg} 3 \mathrm{~A})$ plays a key role in inflammationassociated pancreatic carcinogenesis by promoting cell proliferation, inhibiting apoptosis, and regulating cancer cell migration and invasion. This study aimed to reveal a novel immuno-regulatory mechanism by which Reg3A modulates tumour-promoting responses during pancreatic cancer (PC) progression. In an in vitro Transwell system that allowed the direct co-culture of human peripheral blood-derived dendritic cells (DCs) and Reg3A-overexpressing/ silenced human PC cells, PC cell-derived Reg3A was found to downregulate CD80, CD83 and CD86 expression on educated DCs, increase DC endocytic function, inhibit DC-induced T lymphocyte proliferation, reduce IL-12p70 production, and enhance IL-23 production by DCs. The positive effect of tumourderived Reg3A-educated human DCs on PC progression was demonstrated in vivo by intraperitoneally transferring them into PC-implanted severe combined immunodeficiency (SCID) mice reconstituted with human T cells. A Reg3A-JAK2/STAT3 positive feedback loop was identified in DCs educated with Reg3A. In conclusion, as a tumour-derived factor, Reg3A acted to block the differentiation and maturation of the most important antigen-presenting cells, DCs, causing them to limit their potential anti-tumour responses, thus facilitating PC escape and progression.
\end{abstract}

Keywords: dendritic cells, immunosuppressive tumour microenvironment, inflammation-linked pancreatic carcinogenesis, pancreatic cancer, regenerating islet-derived protein $3 \mathrm{~A}$

\section{INTRODUCTION}

One of the major characteristics of pancreatic cancer (PC) is its highly immunosuppressive tumour microenvironment, which has been recognized as a major contributor to the unique aggressiveness and poor prognosis of this deadly disease (Bazzichetto et al., 2020; Ren et al., 2018). As an integral part of the pancreatic tumour microenvironment, dendritic cells (DCs) act as the most important professional antigen-presenting cells; they have the ability to coordinate multiple components of the immune system, and they play a critical role in directing robust anti-tumour immunity (Deicher et al., 2018; Hegde et al., 2020). However, decreased levels of circulating and tumour-infiltrating DCs have been frequently demonstrated in PC patients, and they have been associated with adverse prognosis and worse survival in patients (Fukunaga et al., 2004; Hirooka et al., 2011; Tjomsland et al., 2010; Yamamoto et al., 2012). Accordingly, DC-based immunotherapy has received increasing attention to control PC progression in recent years (Balachandran et al., 2019; Deicher et al., 2018; Foley et al., 2016; Hegde et al., 2020). Unfortunately, the immunosuppressive tumour microenviron-

Received 27 May, 2021; revised 9 July, 2021; accepted 22 July, 2021; published online 10 September, 2021 
ment in PC poses various challenges that hinder competent DC function, thus impairing the efficacy of DC vaccine immunotherapy (Balachandran et al., 2019; Bazzichetto et al., 2020; Foley et al., 2016; Giovanelli et al., 2019). Blood DCs in PC patients were found to express a semi-mature tolerogenic phenotype, and a correlation between DC maturation status and PC clinical outcome was shown (Fukunaga et al., 2004). Therefore, it is necessary to elucidate the mechanisms of immune tolerance in the PC microenvironment that inhibit DC-triggered anti-tumour immune responses.

Tjomsland et al. (2010) pointed to a role for the inflammatory factors released from the tumour in transforming myeloid and plasmacytoid DCs into semi-mature cells in PC patients. Systemic and local chronic inflammation has long been accepted as one of the most significant risk factors for PC, and the PC-associated inflammatory tumour microenvironment greatly contributes to tumour growth and metastasis (Padoan et al., 2019). Recently, regenerating islet-derived protein 3A (Reg3A), an inflammatory molecule highly expressed in the pancreas, has attracted considerable attention as one of the drivers of inflammation-associated pancreatic carcinogenesis (Liu et al., 2015; Wang et al., 2014; Zhang et al., 2019). During pancreatic inflammation, the secretion of Reg3A by inflamed acinar cells is sensitively and markedly upregulated (Takasawa, 2016; Zhang et al., 2019). Therefore, this molecule was previously named pancreatitis-associated protein (Zhang et al., 2019), and it has long been known as a marker for pancreatic inflammatory disorders (Takasawa, 2016). Accumulating evidence has reported the significantly high expression of Reg3A in samples collected from PC patients, which was closely related to their clinical outcome (Wang et al., 2014; Zhang et al., 2019). Previous studies (Liu et al., 2015; Wang et al., 2014; Zhang et al., 2019) have demonstrated the tumorigenesis effect of Reg3A through its ability to induce cell proliferation, inhibit cell apoptosis, and regulate cancer cell migration and invasion. In PC cells, the activation of Reg3A enhanced the JAK2/STAT3 pathway, which had the ability to directly increase Reg3A expression, thus constituting a positive feedback mechanism to further upregulate Reg3A in cancer cells (Liu et al., 2015). This self-induction of Reg3A in PC cells amplifies its tumorigenesis effect (Liu et al., 2015).

Interestingly, previous in vivo studies (Liu et al., 2017; Yin et al., 2015) found that Reg3g, the mouse homologue of human Reg3A, reduced DC maturation in mice, suggesting a novel mechanism for PC promotion by Reg3g. Therefore, we hypothesized that Reg3A released by PC cells could act as a tumour-derived factor to educate DCs (the most important antigen-presenting cells) and facilitate PC escape and progression by programming an immunosuppressive tumour microenvironment. To test our hypothesis, this study investigated the direct effect of Reg3A silencing or overexpression in human PC cells on the differentiation, function and survival of co-cultured human monocyte-derived DCs using an in vitro transwell system. Then, the effect of tumour-derived Reg3A-educated DCs on PC progression was determined in vivo by intraperitoneal transfer in PC-implanted severe combined immunodeficiency (SCID) mice reconstituted with T cells.

\section{MATERIALS AND METHODS}

\section{Generation of human monocyte-derived DCs}

Monocyte-derived DCs were generated as described previously with minor modifications (Chen et al., 2020). Briefly, human peripheral blood mononuclear cells were isolated from fresh venous blood taken from healthy blood donors using Histopaque-1077 (Sigma-Aldrich, USA) by density gradient centrifugation. CD14 ${ }^{+}$cells were prepared from isolated mononuclear cells by positive selection using CD $14^{+}$microbeads (Miltenyi Biotec, Germany). Then, DCs were generated by culturing for 5 days in RPMl-1640 medium supplemented with $50 \mathrm{ng} / \mathrm{ml}$ recombinant human granulocyte-macrophage colony-stimulating factor (rhGM-CSF) and $50 \mathrm{ng} / \mathrm{ml}$ recombinant human interleukin (rhlL)-4. The purity of the resulting human monocyte-derived DCs was more than $99 \%$, as determined by anti-CD11c staining.

\section{PC cell culture and overexpression/silencing of tumour-de- rived Reg3A}

The human PC cell lines BxPC-3, AsPC-1, SW1990 and PANC1 were purchased from the American Type Culture Collection (ATCC, USA). The cells were maintained in RPMI 1640 medium supplemented with $10 \%$ foetal calf serum and 100 units/ $\mathrm{ml}$ penicillin/streptomycin under a $5 \% \mathrm{CO}_{2}$ environment at $37^{\circ} \mathrm{C}$. Then, AsPC -1 and BxPC -3 cells, which were previously reported to exhibit low constitutive expression of Reg3A (Wang et al., 2014), were transfected with CMV-MCS-3flagREG3A-SV40-neomycin plasmids (Jikai Gene Chemical, China). SW1990 and PANC-1 cells, in which overexpression of Reg3A was previously observed (Wang et al., 2014), were transiently transfected with $50 \mathrm{nmol} / \mathrm{L}$ siRNA-REG3A, the target sequence of which was GCAGTGATGTGATGAATTA (RiboBio, China). Reg3A mRNA and protein expression in $P C$ cells was detected by real-time polymerase chain reaction (PCR) and western blotting, respectively, after $48 \mathrm{~h}$ of transfection (data for Reg3A mRNA and protein expression in PC cells after Reg3A overexpression or silencing are shown in Supplementary Fig. S1). Cells with transfection rates of $60 \%$ $70 \%$ were used for the following experiments.

\section{Co-culture of DCs and Reg3A-overexpressing/Reg3A-si- lenced PC cells in a Transwell system}

Direct co-culture of DCs and PC cells in a non-contact manner was performed using a 6 -well transwell plate $(0.4 \mu \mathrm{m}$ pore size; Corning, UK). PC cells were transfected with empty vector (CMV-MCS-3flag-NEG-SV40-neomycin), Reg3A plasmid (CMV-MCS-3flag-REG3A-SV40-neomycin), siRNA-scramble or siRNA-REG3A and cultured for $48 \mathrm{~h}$ prior to co-culture. Then, DCs and Reg3A-overexpressing/silenced PC cells at a ratio of 1:1 were seeded in the lower and upper chambers of the transwells, respectively, and co-cultured for $48 \mathrm{~h}$. Half of the culture medium was replaced every day.

\section{Analysis of DC phenotype using flow cytometry}

Following a 48-h co-culture with Reg3A-overexpressing/ silenced PC cells, approximately $2 \times 10^{6}$ DCs were collected and stained using PE-anti-CD80, APC-anti-CD86 and Pe-Cy7anti-CD83 antibodies (BioLegend, USA). The expression of 
these surface molecules was analysed using a flow cytometer (CytoFLEX S; Beckman, USA).

\section{DC endocytosis assay using fluorescein isothiocyanate (FITC)-dextran uptake}

The ability of DCs to take up antigen was evaluated by incubation of $5 \times 10^{4} \mathrm{DCs}$ collected from the co-culture system with $1 \mathrm{mg} / \mathrm{ml} \mathrm{FITC-dextran} \mathrm{(Sigma-Aldrich)} \mathrm{at} 37^{\circ} \mathrm{C}$ for $1 \mathrm{~h}$. A control incubated at $4^{\circ} \mathrm{C}$ was used to exclude the extracellular binding of FITC-dextran. DCs were then washed in cold fluorescence-activated cell sorting (FACS) buffer and analysed by flow cytometry.

\section{Mixed lymphocyte reaction (MLR)}

The collected DCs served as stimulator cells, and T lymphocytes served as responder cells in the MLR. T lymphocytes were obtained by passing the isolated human peripheral blood mononuclear cells through a nylon wool column, and then they were co-cultured with the collected DCs in a $5 \%$ $\mathrm{CO}_{2}$ humidified environment at $37^{\circ} \mathrm{C}$ for $96 \mathrm{~h}$. The ratio of stimulators to responders was 1:10. A cell counting kit-8 assay was conducted to detect $T$ lymphocyte proliferation. The ability of DCs to interact with proliferating T lymphocytes was assessed by measuring the absorbance at $450 \mathrm{~nm}$, which was then normalized to that of the Con A-positive control, producing a ratio.

\section{ELISAs to measure the levels of interleukin (IL)-12p70 and IL-23 produced by DCs}

After $48 \mathrm{~h}$ of co-culture, the cell-free supernatants were collected from the PC-DC co-culture system. The concentrations of IL-12p70 and IL-23 in the supernatants were detected using ELISA kits according to the manufacturer's instructions (Abcam, UK).

\section{Real-time PCR}

Total RNA was isolated from DCs collected from the co-culture system using the TRIzol reagent (Invitrogen, USA). The mRNA expression levels of STAT3, JAK2, and Reg3A in DCs were measured by real-time PCR. The primer sequences were as previously described (Liu et al., 2015). The relative amount of gene expression was normalized according to $\beta$-actin.

\section{Western blots}

The protein expression levels of STAT3, JAK2, and Reg3A in collected DCs were measured by western blots. Protein extracted from DCs using RIPA protein lysate (Shanghai Beyotime Biotechnology, China) was separated on a $12 \%$ sodium dodecyl sulphate-polyacrylamide gel by electrophoresis and then electroblotted onto a nitrocellulose membrane. The membrane was incubated with primary antibodies against JAK2, STAT3, pSTAT3 (Cell Signaling Technology, USA), Reg3A (Absin, China), and $\beta$-actin (Sungene, China). Protein levels were quantified based on the band intensities following normalization to $\beta$-actin.

\section{PC cell cycle assay}

After $48 \mathrm{~h}$ of co-culture, PC cells co-cultured with DCs were collected, stained with propidium iodide (PI) for 30 min at $4^{\circ} \mathrm{C}$, and then immediately analysed by flow cytometry.

\section{Cell apoptosis assay}

After $48 \mathrm{~h}$ of co-culture, PC cells co-cultured with DCs were collected from the transwell system and stained with Annexin V-FITC and PI for apoptosis detection by flow cytometry.

\section{In vivo tumorigenesis}

Human PC BxPC-3 cells $\left(5 \times 10^{6}\right)$ were implanted subcutaneously onto the backs of 4-week-old female C.B17SCID mice (Charles River, China). Meanwhile, isolated human peripheral blood T lymphocytes $\left(4 \times 10^{7}\right)$ were intraperitoneally injected into the mice. Four weeks later, immune reconstituted mice bearing palpable tumours were transferred $1 \times 10^{6}$ human DCs that were previously co-cultured with BxPC-3 cells transfected with either CMV-MCS-3flag-REG3A-SV40-neomycin or CMV-MCS-3flag-NEG-SV40-neomycin for $48 \mathrm{~h}$. On weeks 5 and 6 , transfer was repeated once a week. On week 7 . mice were sacrificed. Tumour volume was determined once a week by measuring the minimum width (a) and ribbon width (b) of the tumour, and then calculated by the formula $V=a b^{2} / 2$. The tumour tissues were collected and weighed. Peripheral blood samples were collected by quickly removing the eyeballs. After incubation with RBC lysis buffer to remove red blood cells, the samples were mixed with anti-human CD4-FITC (Absin) and anti-human CD8-PE (BD Pharmingen, USA). $C D 4^{+}$and $C D 8^{+}$cell populations were evaluated using flow cytometry.

\section{Statistical analysis}

All experiments were conducted with three replicates. Data are expressed as the mean \pm SD and were analysed by oneway ANOVA with a post-hoc Tukey test using IBM SPSS Statistics (ver. 24.0; IBM, USA). $P<0.05$ was considered an indication of statistical significance.

\section{RESULTS}

\section{Tumour-derived Reg3A-educated DCs exhibited a more immature phenotype}

We first examined the maturation status of DCs co-cultured with Reg3A-overexpressing/Reg3A-silenced PC cells by analysing expression of mature DC surface markers. As shown in Fig. 1, after a 48-h co-culture of Reg3A-overexpressing AsPC1 and BxPC-3 cells, DCs expressed lower levels of CD80, CD83, and CD86 compared to DCs co-cultured with control AsPC-1 and BxPC-3 cells. However, the expression levels of these surface molecules in DCs co-cultured with Reg3A-silenced SW1990 and PANC-1 cells were higher than in the controls. This finding suggested that tumour-derived Reg3A reduced the maturation of DCs, and they tended to maintain their immature tolerogenic phenotype.

Tumour-derived Reg3A-educated DCs showed improved endocytosis and impaired T lymphocyte stimulatory function

One of the major functional characteristics of immature DCs is their high endocytic ability, by which they effectively capture antigens (Liu et al., 2017; Yin et al., 2015). As shown in 
Reg3A and Inflammation-Linked Pancreatic Carcinogenesis Jie Guo et al.

A

Co-cultured with BXPC-3REG3Ahigh cells
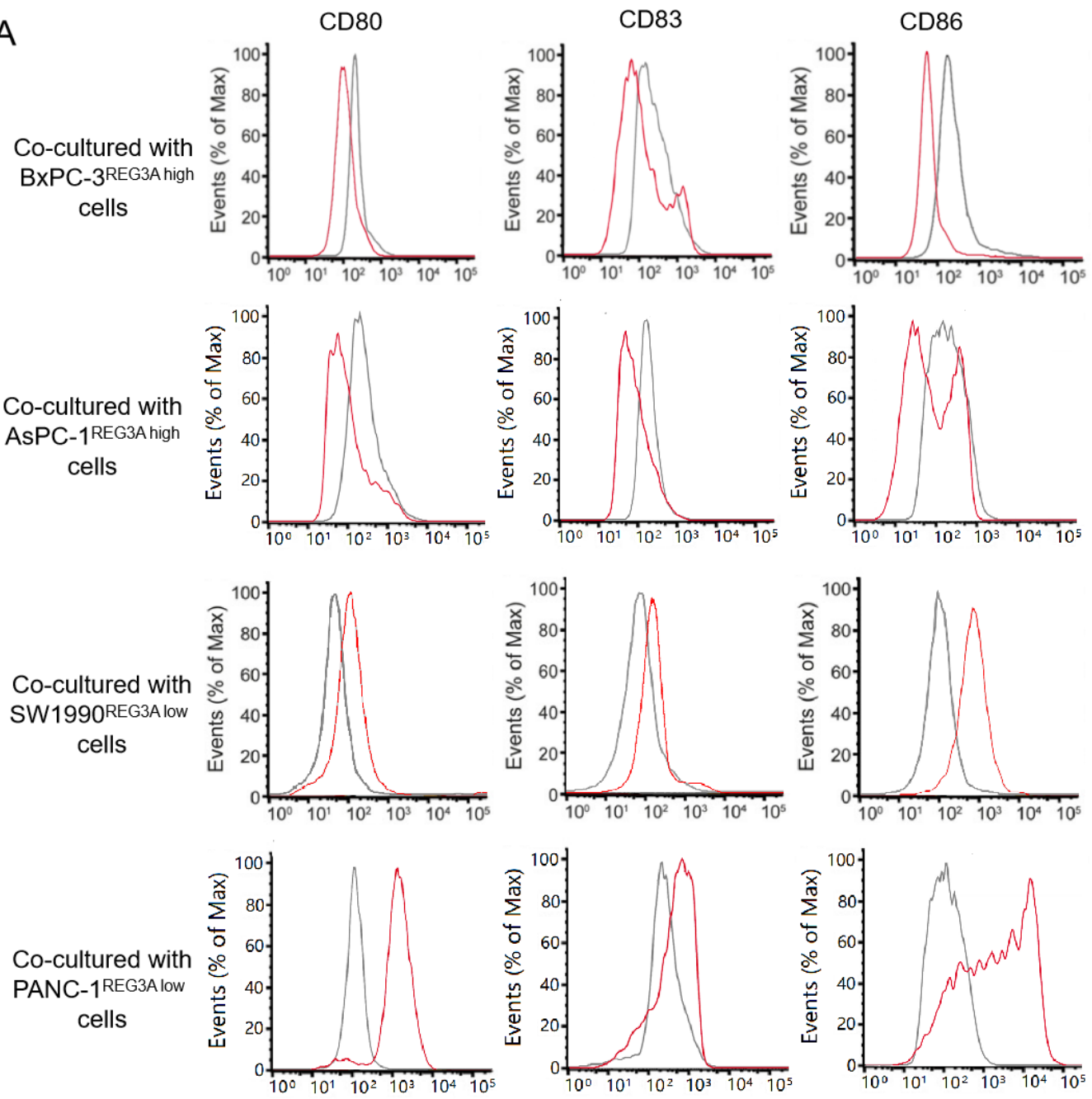

$\mathrm{B}=\mathrm{DCs}$ co-cultured with control PC cells DCs co-cultured with Reg3A overexpressed PC cells

$\square$ DCs co-cultured with control PC cells

$\square$ DCs co-cultured with Reg3A silenced PC cells

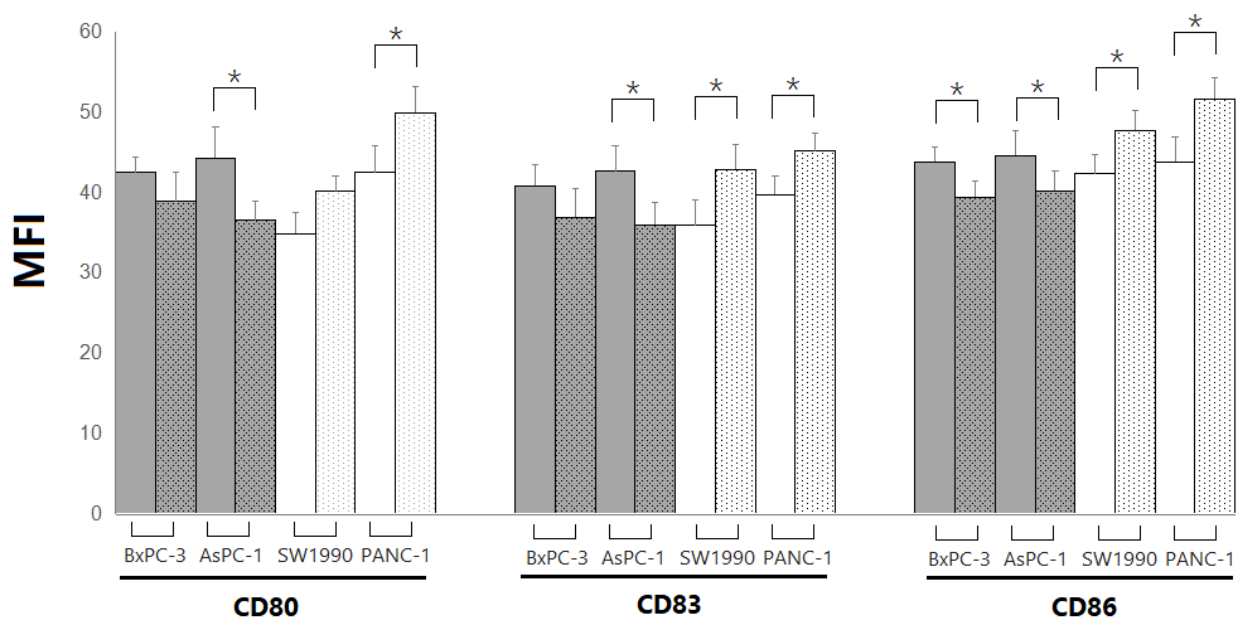

Fig. 1. Effect of tumour-derived Reg3A on the phenotype of DCs. The expression of CD80, CD83, and CD86 on DCs was measured by flow cytometry. (A) Representative histograms. Red line: The expression of CD80, CD83, and CD86 on DCs co-cultured with Reg3Aoverexpressing/silenced PC cells. Grey line: The expression of CD80, CD83, and CD86 on DCs co-cultured with PC cells transfected with mock vector. (B) Bar graph represents the statistically transformed data. Data are expressed as the mean fluorescence intensity $(M F I) . n=3$, $\star P<0.05$, compared with the corresponding control group. Data represent three independent experiments. 
A

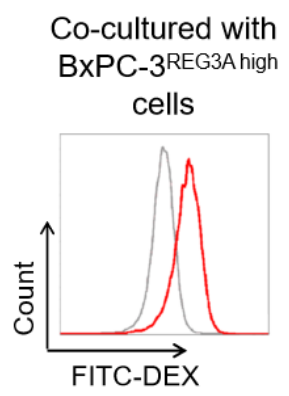

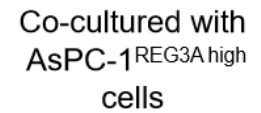

cells

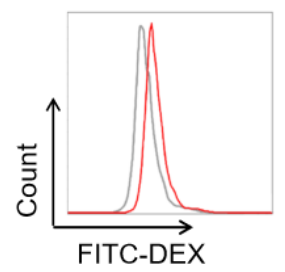

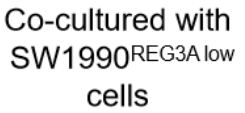

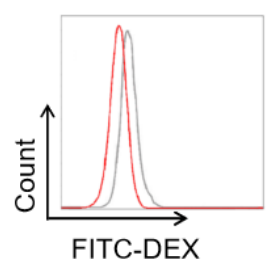

Co-cultured with

PANC-1 REG3Alow cells

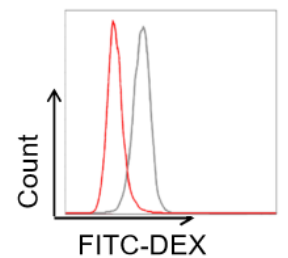

\section{nCs co-cultured with control PC cells \\ DCs co-cultured with Reg3A overexpressed PC cells}
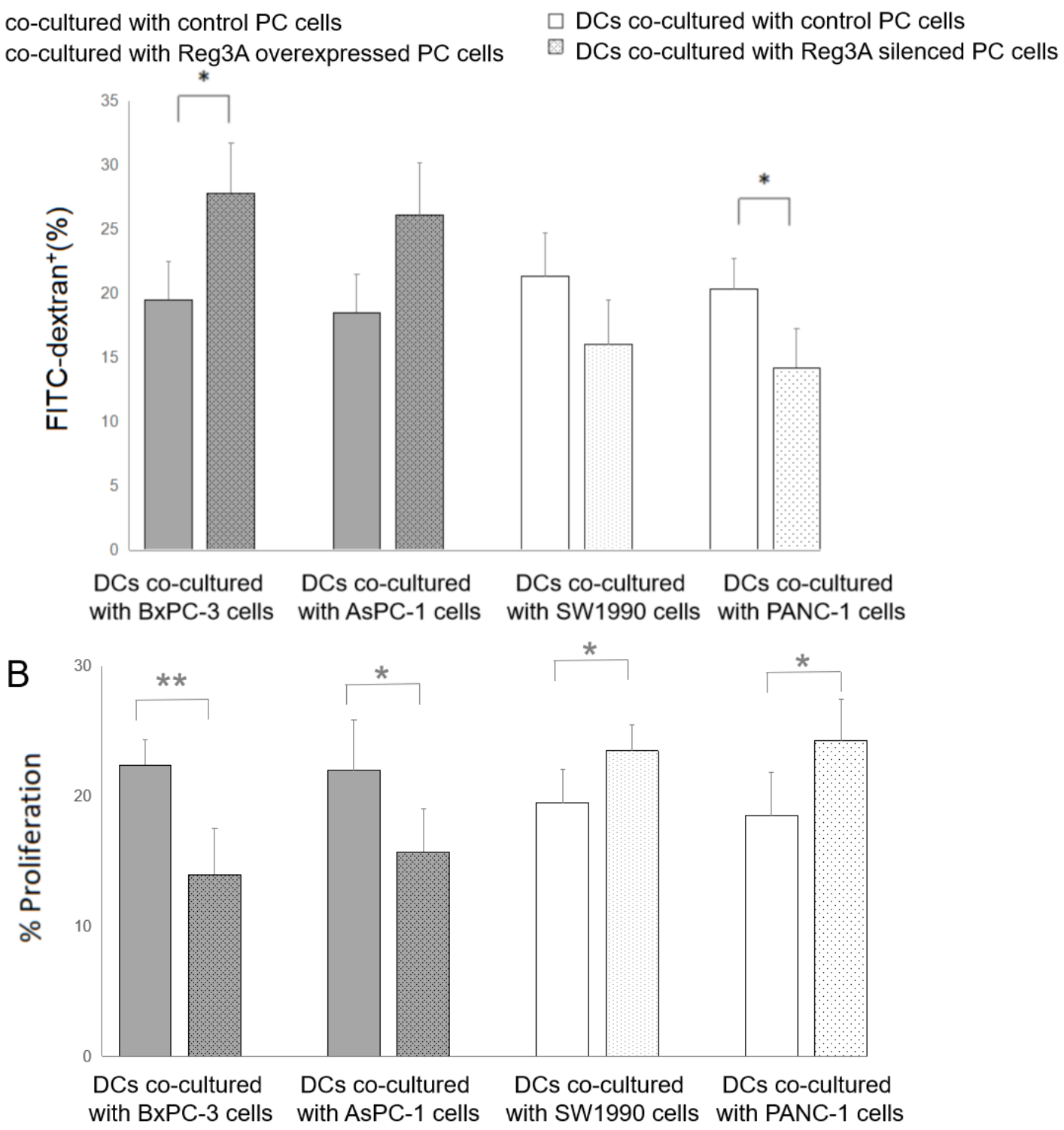

Fig. 2. Effect of tumour-derived Reg3A on the function of DCs. (A) Uptake of FITC-dextran by DCs was measured by flow cytometry. Red line: The endocytic function of DCs co-cultured with Reg3A-overexpressing/silenced PC cells. Grey line: The endocytic function of DCs co-cultured with PC cells transfected with mock vector. (B) The ability of DCs to induce T lymphocyte proliferation was assessed by measuring the absorbance at $450 \mathrm{~nm}$, which was normalized to that of the Con A-positive control producing a ratio. $\mathrm{n}=3, * P<0.05$, $* * P<0.01$, compared with the corresponding control group. Data represent three independent experiments. 
Fig. 2A, we found that the co-culture with BxPC-3 ${ }^{\mathrm{REG} 3 \mathrm{~A}}$ high and $A x P C-1^{\text {REG }}$ Aigh cells drove DCs to internalize antigens more efficiently, while DCs co-cultured with SW1990 ${ }^{\text {REG3A }}$ low and PANC-1 ${ }^{\text {REG3A low }}$ cells showed decreased endocytosis compared to those co-cultured with control PC cells. Furthermore, Reg3A released from BxPC $-3^{\text {REG } 3 A \text { high }}$ and $A \times P C-1^{\text {REG } 3 A}$ high cells suppressed the ability of co-cultured DCs to stimulate $\mathrm{T}$ lymphocytes, as assessed by MLRs, with reductions of $37.7 \%$ and $28.6 \%$ in T cell activation, respectively, compared with the controls $(P<0.05, P<0.01)$ (Fig. 2B). However, DCs co-cultured with SW1990 REG3A low and PANC-1 ${ }^{\text {REG3A low }}$ cells stimulated $\mathrm{T}$ lymphocyte proliferation significantly more compared to DCs co-cultured with control SW1990 and PANC-1 cells $(P<0.05)$ (Fig. 2B). The percentages of proliferative $T$ lymphocytes stimulated with DCs co-cultured with SW1990 ${ }^{\text {REG3A low }}$ and PANC-1 ${ }^{\text {REG3A low }}$ cells were enhanced by
$20.6 \%$ and $31.2 \%$, respectively, compared with T cells stimulated with DCs co-cultured with the corresponding control PC cells.

\section{Tumour-derived Reg3A-educated DCs secreted less IL- $12 \mathrm{p} 70$ but more IL-23}

To further confirm the inhibitory effect of tumour-derived Reg3A on DC maturation, we measured the levels of IL12 p70 and IL-23, two representative immunostimulatory cytokines that are generally accepted to be mainly secreted by mature DCs (Chen et al., 2016; Dudek et al., 2013), in the supernatants collected from the PC-DC co-culture system. As shown in Fig. 3, IL-12p70 production by DCs was significantly suppressed when they were co-cultured with Reg3A-overexpressing BxPC-3 and AxPC-1 cells and was markedly enhanced when they were co-cultured with Reg3A-silenced

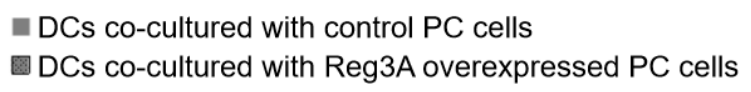

$\square$ DCs co-cultured with control PC cells

$\square$ DCs co-cultured with Reg3A silenced PC cells
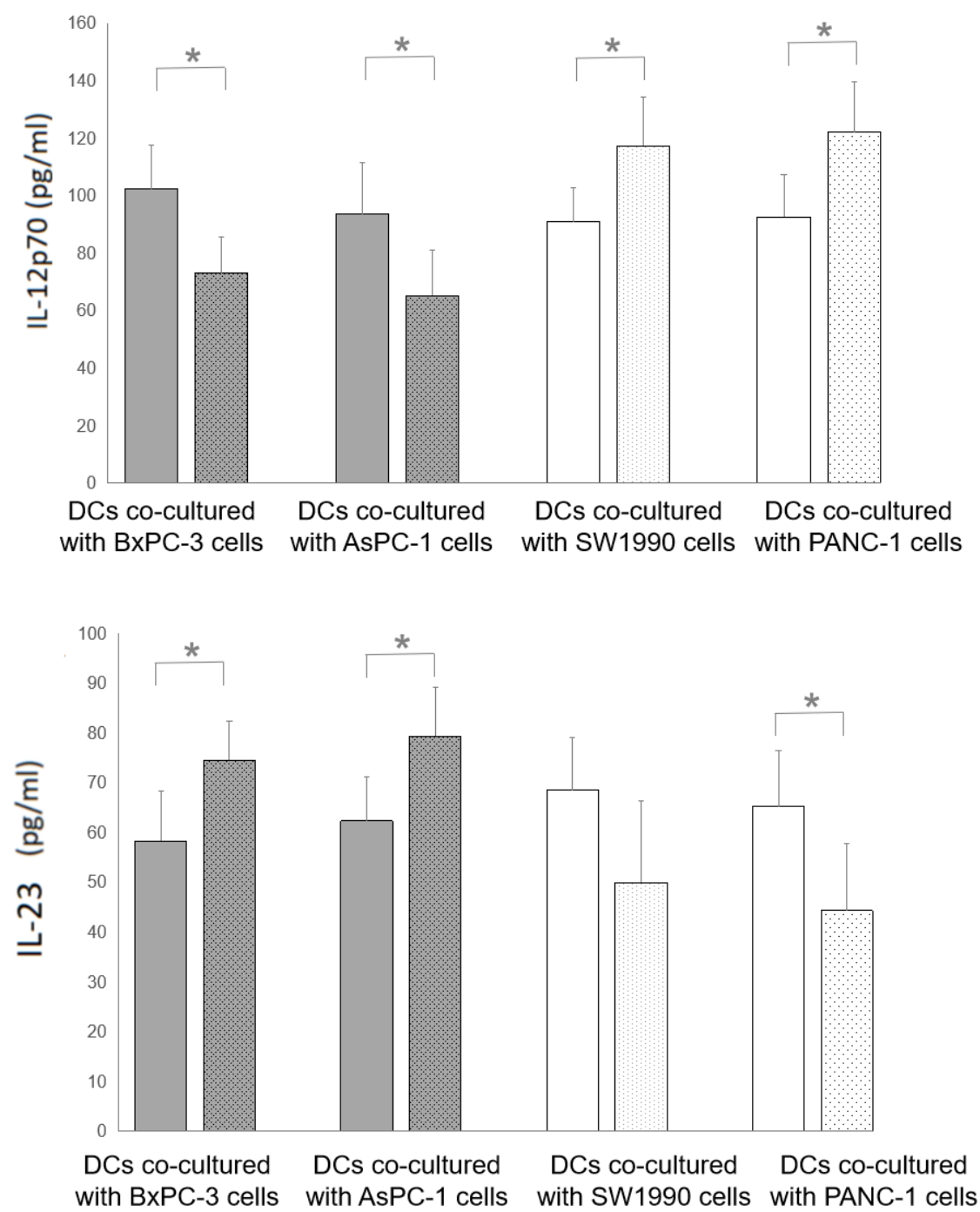

Fig. 3. Effect of tumour-derived Reg3A on the levels of IL-12p70 and IL-23 in supernatants from the PC-DC co-culture system. $n=3$, $\star P<0.05$, compared with the corresponding control group. Data represent three independent experiments. 
SW1990 and PANC-1 cells, as compared to DCs co-cultured with the corresponding PC cells $(P<0.05)$. Unexpectedly, co-culturing with Reg3A-overexpressing PC cells increased the IL-23 levels in the transwell system $(P<0.05)$, while DCs co-cultured with PANC-1 $1^{\text {REG }}$ low cells secreted less IL-23 than those co-cultured with control PANC-1 cells $(P<0.05)$.

\section{A Reg3A-JAK2/STAT3 positive feedback loop might be involved in the effect of tumour-derived Reg3A on co-cul- tured DCs}

A previous study (Liu et al., 2015) demonstrated that Reg3A enhances the JAK2/STAT3 pathway and further increases Reg3A expression in PC cells, which results in a Reg3A-JAK2/ STAT3 positive feedback loop leading to the amplification of the oncogenic effects of Reg3A. Coincidentally, JAK2/STAT3 signalling has been considered to play a critical role in the negative regulation of DC activation and maturation (Gabrilovich, 2004; Nefedova et al., 2005). Therefore, we measured the mRNA and protein expression of STAT3, JAK2, and Reg3A in DCs co-cultured with PCs. As shown in Fig. 4A, the mRNA expression levels of STAT3, JAK2, and Reg3A in DCs co-cultured with Reg3A-overexpressing PC cells were 3.0to 3.7-fold higher, 1.9- to 2.8-fold higher, and 1.9- to 2.6fold higher, respectively, than those in DCs co-cultured with control PC cells $(P<0.05, P<0.01)$. However, STAT3, JAK2, and Reg3A mRNA expression in DCs co-cultured with Reg3A-silenced PC cells was 38\%-59\%, 43\%-58\%, and 26\%$43 \%$ of that in the controls, respectively $(P<0.05, P<0.01)$. Western blot analysis confirmed the upregulation of STAT3, pSTAT3, JAK2, and Reg3A expression at the protein level in DCs educated by tumour-derived Reg3A (Fig. 4B).

\section{Tumour-derived Reg3A-educated DCs promoted PC pro- gression}

To explore the effect of tumour-derived Reg3A-educated DCs on PC progression, we first collected PC cells co-cultured with DCs in a transwell system, and then measured their apoptosis and cell cycle progression. As shown in Supplementary Figs. S2 and S3, the transfection of the Reg3A overexpression plasmid or siRNA-REG3A significantly affected the apoptosis rates and the percentages of cells in the $S$ phases $(P<0.05$, $P<0.01$ ), which was in line with previous studies reporting the anti-apoptosis and proliferation-inducing activities of Reg3A (Liu et al., 2015; Wang et al., 2014; Zhang et al., 2019). However, no significant difference in PC cell apoptosis or cycle progression was found between pre- and post-coculture with Reg3A-educated DCs. This finding suggested that tumour-derived Reg3A-educated DCs appeared to have no direct in vitro effect on PC cell survival and growth in the co-culture system.

We next evaluated the in vivo effect of tumour-derived Reg3A-educated DCs on PC progression by intraperitoneal transfer of DCs into BxPC-3-implanted SCID mice reconstituted with human T cells. As the time of BxPC-3 implantation increased, a progressive increase in tumour volume was shown in both groups, but the growth rates in the mice transferred tumour-derived Reg3A-educated DCs were further enhanced compared to those transferred control DCs. After 7 weeks of BxPC-3 implantation, the tumours were dramatically larger and heavier in animals transferred tumour-derived Reg3A-educated DCs $(P<0.05)$ (Figs. 5A-5C). Flow cytometry analysis of the peripheral blood samples from sacrificed mice revealed that the percentages of human $C D 4^{+}$and $C D 8^{+} T$ cells in mice transferred with tumour-derived Reg3A-educated DCs were $40 \%$ and $66 \%$, respectively, of those in mice transferred with control DCs $(P<0.05)$ (Figs. 5D and 5E).

\section{DISCUSSION}

The presence of DCs in the tumour microenvironment has been considered a double-edged sword, as they may not only initiate potent anti-tumour immune responses but also block anti-tumour immunity and stimulate cancer cell growth (Conejo-Garcia et al., 2016; Gabrilovich, 2004; Nefedova et al., 2005). As a specific tumour microenvironment signalling pathway, multiple tumour-derived factors, including vascular endothelial growth factor, macrophage colony stimulating factor, TGF- $\beta$, IL-6, and IL-10, among others (Conejo-Garcia et al., 2016; Gabrilovich, 2004; Ma et al., 2020), have been demonstrated to be consistently released by tumour cells; these factors mediate the DC dysfunction in cancer by inhibiting DC recruitment and activation, which results in non-responsiveness to tumours. However, the list of tumour-derived factors that modulate DC defects in cancer is constantly growing (Gabrilovich, 2004). In particular, increasing interest has been focused on inflammatory factors derived from PC tumours as targets for the control of inflammation-linked pancreatic carcinogenesis (Tjomsland et al., 2010; Zhang et al., 2019). In the present study, we demonstrated that a secreted protein, Reg3A (which is specifically highly produced by inflamed and malignant pancreatic tissues), continuously released by PC cells could act as a tumour-derived factor to educate DCs (potent antigen-presenting cells), thus contributing to the immunosuppressive tumour microenvironment and facilitating inflammation-linked pancreatic carcinogenesis.

Yin et al. (2015) reported that Reg3g overexpression in C57BL/6 mice significantly accelerated and exacerbated the progression from caerulein-induced chronic pancreatitis to the formation of recognizable PC tumours in the pancreas. Here, we used a transwell system allowing the in vitro direct co-culture of human DCs and Reg3A-overexpressing/Reg3A-silenced PC cells in vitro and demonstrated that Reg3A derived from PC cells blocked the differentiation of resting/ immature tolerogenic DCs into activated/mature immunogenic DCs, thus inducing DCs to restrain their potential anti-tumour responses. Activated/mature DCs are characterized by enhanced expression of co-stimulatory molecules, which enable them to interact with naive T cells, increase their capacity to activate $T$ cells, reduce their antigen uptake, and decrease their production of cytokines such as IL12 and IL-23 (Chen et al., 2016; Deicher et al., 2018; Dudek et al., 2013; Hegde et al., 2020). Our results showed that tumour-derived Reg3A decreased the expression of CD80, CD83 and CD86 on DCs, increased the endocytic function of DCs, inhibited DC-induced T lymphocyte proliferation and reduced IL-12p70 production by DCs. However, the IL-23 production of Reg3A-educated DCs was unexpectedly increased 
A

$\square$ DCs co-cultured with BxPC-3 ${ }^{\text {control }}$ cells

DCs co-cultured with BxPC-3 ${ }^{\text {REG3A high }}$ cells

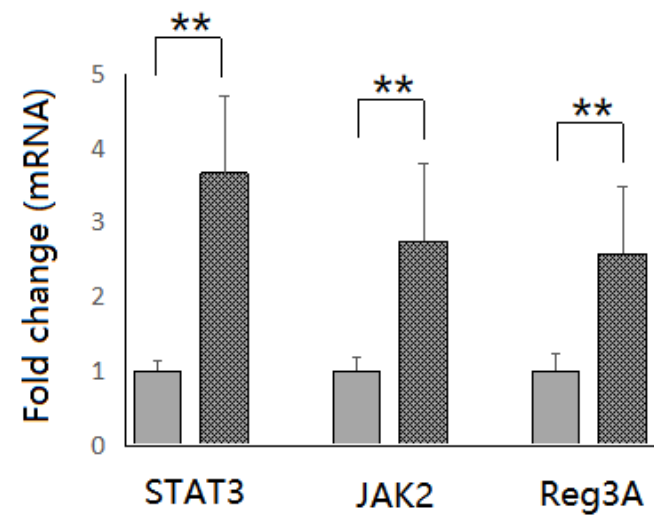

口 DCs co-cultured with SW1990 control cells

$\square$ DCs co-cultured with SW1990 REG3A low cells

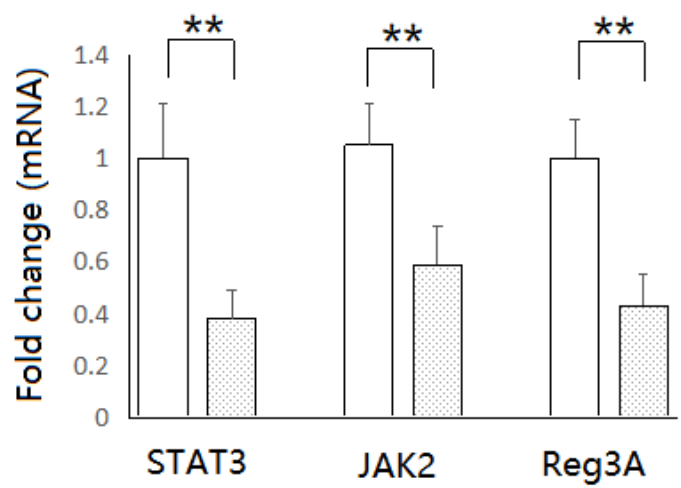

$\square$ DCs co-cultured with AxPC-1 control cells

圈 DCs co-cultured with AxPC-1 ${ }^{\text {REG3A high }}$ cells

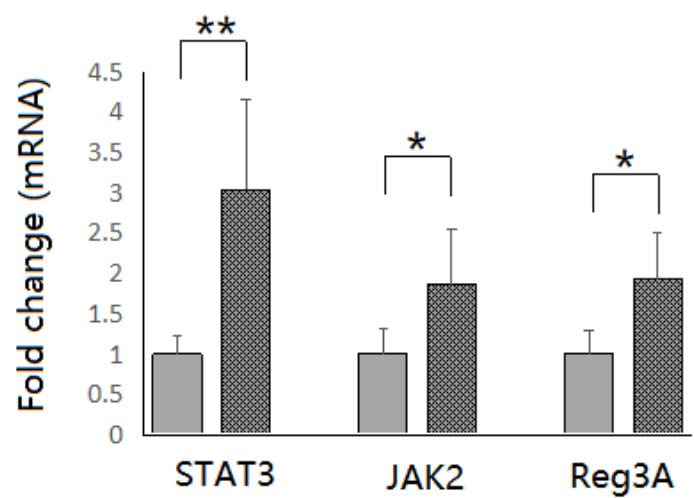

$\square$ DCs co-cultured with PANC-1 ${ }^{\text {control }}$ cells

$\square$ DCs co-cultured with PANC-1 ${ }^{\text {REG3A low }}$ cells

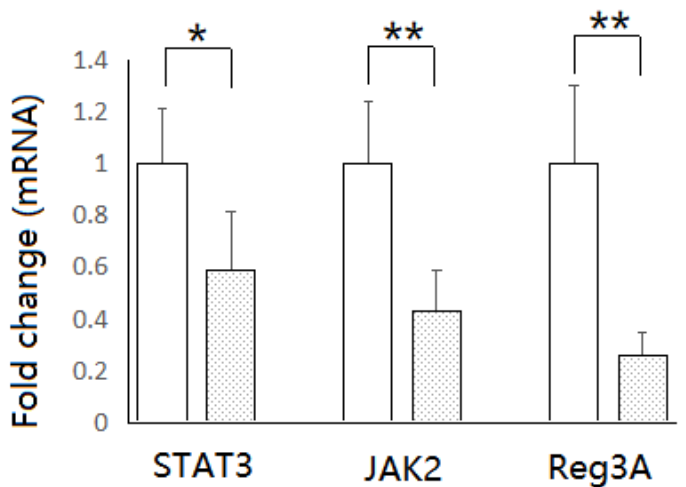

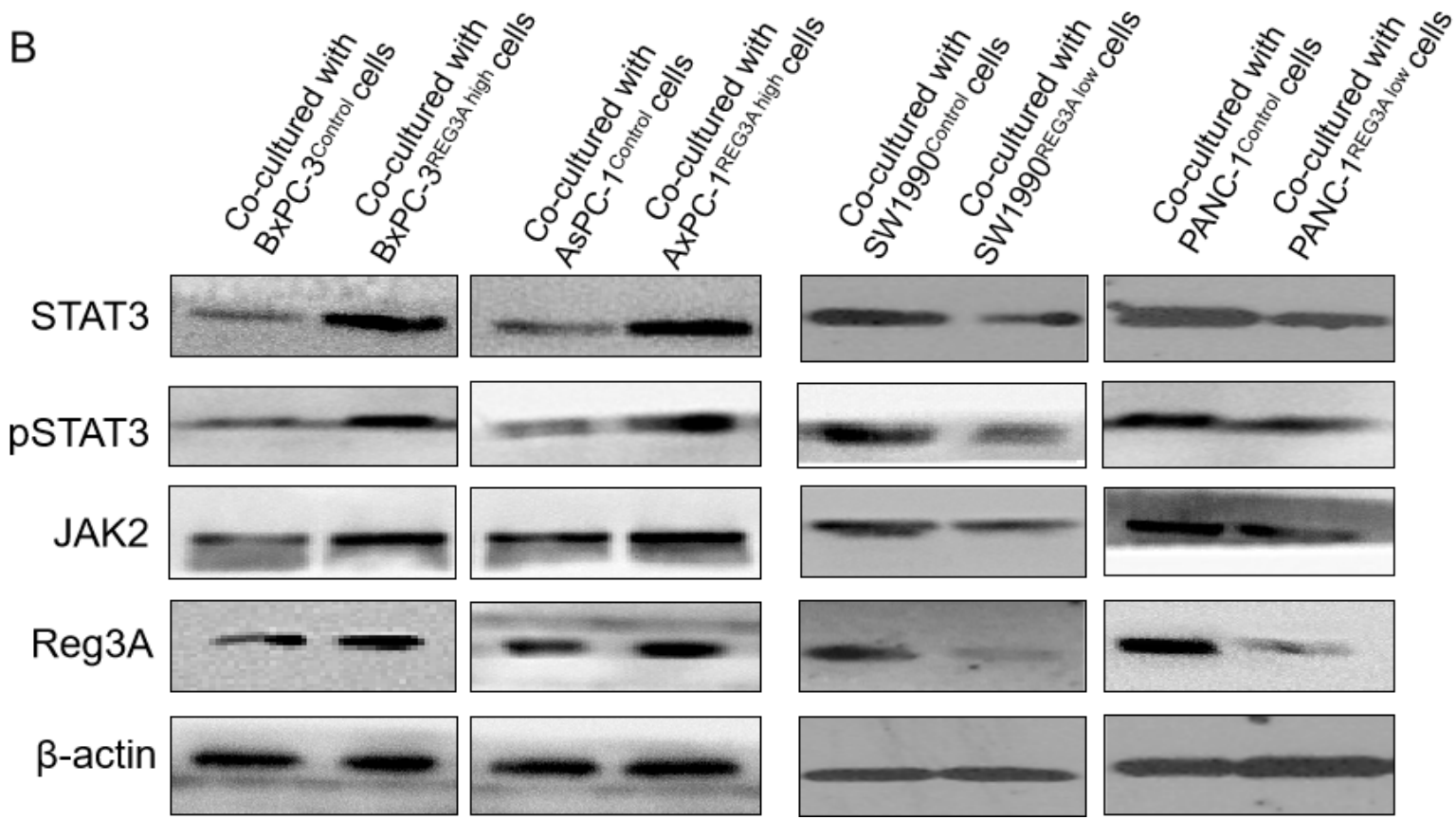

Fig. 4. Effect of tumour-derived Reg3A on STAT3, JAK2 and Reg3A expression in DCs. (A) The mRNA expression levels of STAT3, JAK2 and Reg3A in DCs were determined by real-time PCR. $n=3, * P<0.05, * * P<0.01$, compared with the corresponding control group. (B) The protein expression of STAT3 in DCs was determined by western blot. Data represent three independent experiments. 

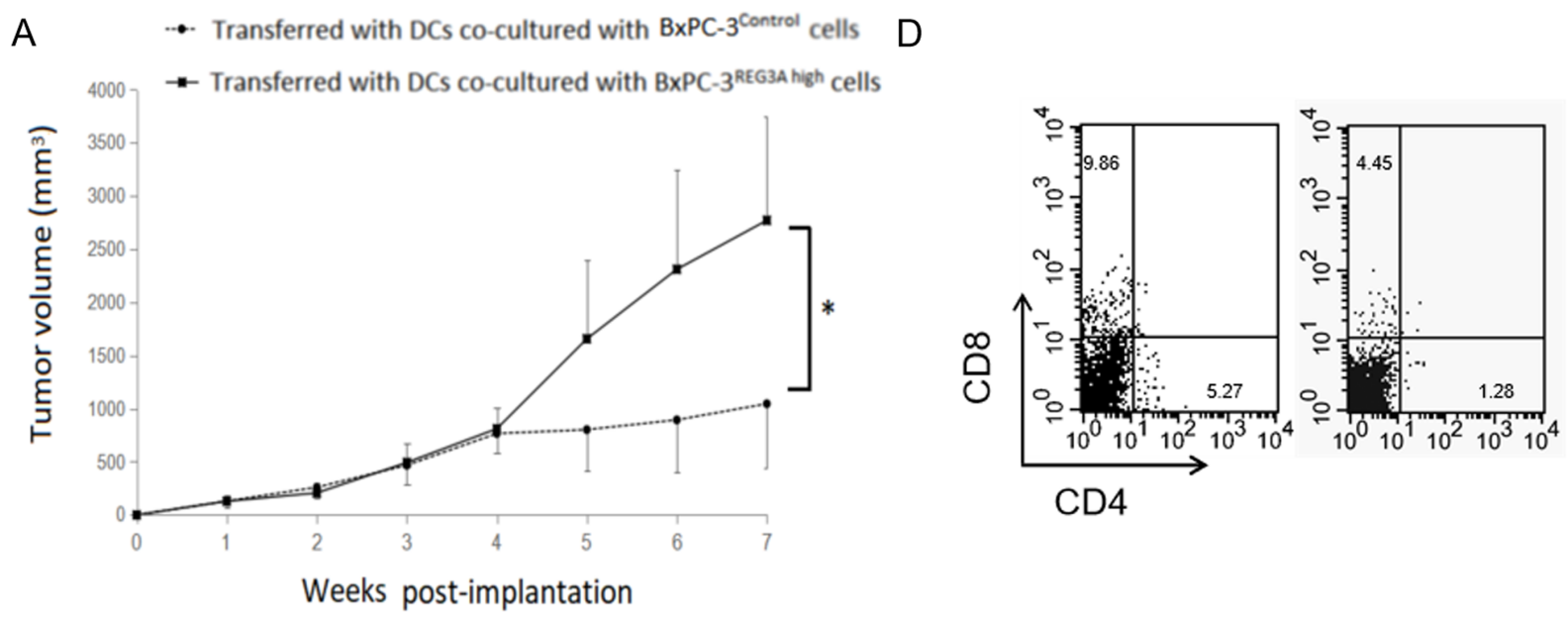

B

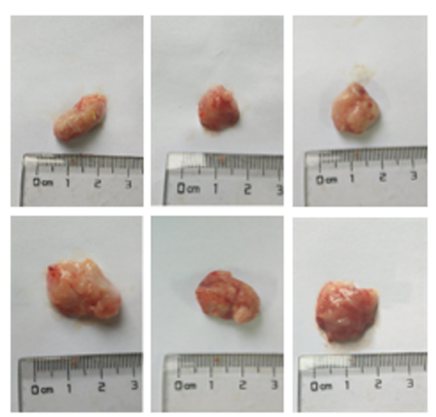

C Transferred with DCs co-cultured with BxPC-3 ${ }^{\text {Control }}$ cells

Transferred with DCs co-cultured with BXPC-3 ${ }^{\text {REG3A high }}$ cells

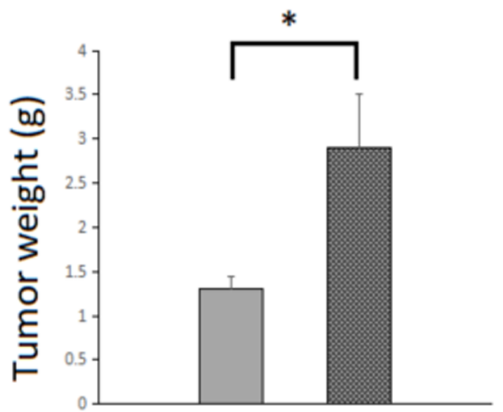

E

Transferred with DCs co-cultured with BxPC-3 $3^{\text {Control }}$ cells
Transferred with DCs co-cultured with BxPC-3 $3^{\text {REG } 3 A \text { high }}$ cells
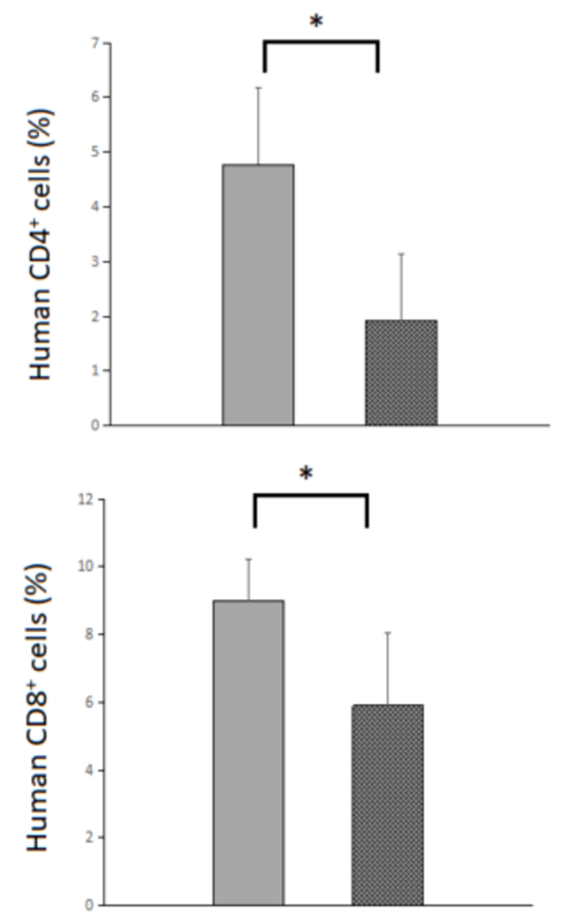

Fig. 5. Effect of tumour-derived Reg3A-educated DCs on the growth of BxPC-3 tumour xenografts and the percentage of human $\mathrm{CD}^{+}$and $\mathrm{CD}^{+} \mathrm{T}$ cells in human immune reconstituted SCID mice. (A) Tumour volume was determined once a week by measuring the minimum width (a) and ribbon width (b) of the tumour and calculated by the formula $V=a b^{2} / 2$. (B) Images of dissected tumours from mice transferred BXPC-3 $3^{\text {control }}$ (upper) and BXPC-3 ${ }^{\text {REG3A high }}$ (lower) cells. (C) Tumour weights. (D and E) Percentages of cells positive for human $\mathrm{CD}^{+}$and $\mathrm{CD} 8^{+} \mathrm{T}$ cells in peripheral blood samples. $\mathrm{n}=3, * P<0.05$, compared with the mice transferred BxPC-3 $3^{\text {Control }}$ cells. Data represent three independent experiments.

compared to that of control DCs. IL-12p70 and IL-23 are selectively secreted by activated antigen-presenting cells, such as DCs, but are not produced by PC cells (Chen et al., 2016; Dudek et al., 2013). Thus, the levels of these two cytokines in the PC-DC co-culture system were measured to confirm the effect of PC cell-derived Reg3A on DC maturation. Nevertheless, accumulating evidence has shown the conflicting roles of IL-12 and IL-23 in cancer (Chyuan and Lai, 2020; Yan et al., 2018). IL-12 triggers T helper (Th) 1 responses and is generally believed to exert anti-tumour efficacy via downstream activation of interferon- $\gamma$, while overactivated IL-23 signalling drives aberrant Th17 immune responses and has a tumour-promoting function. A clinical study (He et al., 2011) reported that the serum levels of $\mathrm{IL}-23$ in $\mathrm{PC}$ patients were 
2.8-fold higher than those of healthy volunteers and associated with the invasion and metastasis of PC. Therefore, we concluded that tumour-derived Reg3A educated DCs to reduce anti-tumour IL-12 production and enhance tumour-promoting IL-23 secretion, which might at least partly contribute to $\mathrm{PC}$ progression.

Regarding the mechanisms, we found that tumour-derived Reg3A upregulated the expression of STAT3/pSTAT3 and JAK2 in educated DCs. The inhibitory activity of STAT3/ JAK2 on the differentiation and maturity of DCs has been well accepted (Gabrilovich, 2004; Nefedova et al., 2005). Interestingly, similar to that in PC cells (Liu et al., 2015), a Reg3A-JAK2/STAT3 positive feedback loop was also observed in DCs educated with Reg3A, which might maintain and amply the educational effect of tumour-derived Reg3A on DCs. Further studies are needed to identify the direct effect of Reg3A upregulation in DCs on the decreased maturation, antigen uptake, and T cell priming of DCs induced by tumour-derived Reg3A.

After transfer into SCID mice bearing BxPC-3 cells, DCs co-cultured with Reg3A-overexpressing BxPC-3 cells induced significantly more tumour growth compared to control DCs, suggesting that tumour-derived Reg3A-educated DCs had a pro-tumour effect in vivo. However, in the in vitro co-culture system, tumour-derived Reg3A-educated DCs had no significant direct effect on PC cell survival and growth. A possible explanation may be that downstream effector cells required for DC-mediated immunity were not present within the in vitro transwell system. As potent initiators of the immune response, DCs need to prime and activate downstream immune cells, especially $T$ lymphocytes, thereby eliciting the immune response (Deicher et al., 2018; Hegde et al., 2020). Accordingly, there were significantly fewer $C D 4^{+}$and $C D 8^{+}$ $T$ cells in mice transferred tumour-derived Reg3A-educated DCs compared to mice transferred control DCs. However, whether the tumour-derived Reg3A-educated DCs mediate their tumour-promoting effect through the direct attenuation of $\mathrm{T}$ cell proliferation and effector function or through the induction of immune cells with immuno-modulatory function, such as Treg cells, merits further investigation.

In conclusion, our data reveal a novel immuno-regulatory mechanism by which Reg3A, an inflammatory protein highly secreted by inflamed pancreatic acinar cells and PC cells, modulates tumour-promoting responses during PC progression. In a transwell co-cultured system, tumour-derived Reg3A was found to downregulate the expression of CD80, CD83 and CD86 on educated DCs, increase the endocytic function of DCs, inhibit DC-induced T lymphocyte proliferation, and reduce IL-12p70 production but enhance IL-23 production by DCs. The in vivo positive effect of tumour-derived Reg3A-educated DCs on PC progression was demonstrated by intraperitoneally transferring them into PC-implanted SCID mice reconstituted with human T cells. A Reg3A-JAK2/STAT3 feedback loop-associated mechanism appeared to underlie the effect of tumour-derived Reg3A on educated DCs.

Note: Supplementary information is available on the Molecules and Cells website (www. molcells.org).

\section{ACKNOWLEDGMENTS}

This work was funded by grants from the National Natural Science Foundation of China $(81602108,71974153)$ and the Provincial College Students' Innovative Entrepreneurial Training Program in Hubei (201810488115).

\section{AUTHOR CONTRIBUTIONS}

J.W. conceived and designed the experiments. J.G., M.L., and X.H. performed the experiments. J.G., M.L., X.H., and J.W. analysed and interpreted the data. All authors read and approved the final manuscript.

\section{CONFLICT OF INTEREST}

The authors have no potential conflicts of interest to disclose.

ORCID

Jie Guo

Mengfan Liao

Xianmin $\mathrm{Hu}$

Jun Wang

\section{REFERENCES}

Balachandran, V.P., Beatty, G.L., and Dougan, S.K. (2019). Broadening the impact of immunotherapy to pancreatic cancer: challenges and opportunities. Gastroenterology 156, 2056-2072.

Bazzichetto, C., Conciatori, F., Luchini, C., Simionato, F., Santoro, R., Vaccaro, V., Corbo, V., Falcone, I., Ferretti, G., Cognetti, F., et al. (2020). From genetic alterations to tumor microenvironment: the Ariadne's string in pancreatic cancer. Cells 9, 309.

Chen, G., Le, Y., Zhou, L., Gong, L., Li, X., Li, Y., Liao, Q., Duan, K., Tong, J., and Ouyang, W. (2016). Dexmedetomidine inhibits maturation and function of human cord blood-derived dendritic cells by interfering with synthesis and secretion of IL-12 and IL-23. PLoS One 11, e0153288.

Chen, I.L., Todd, I., Tighe, P.J., and Fairclough, L.C. (2020). Electronic cigarette vapour moderately stimulates pro-inflammatory signalling pathways and interleukin- 6 production by human monocyte-derived dendritic cells. Arch. Toxicol. 94, 2097-2112.

Chyuan, I.T. and Lai, J.H. (2020). New insights into the IL-12 and IL23: from a molecular basis to clinical application in immune-mediated inflammation and cancers. Biochem. Pharmacol. 175, 113928.

Conejo-Garcia, J.R., Rutkowski, M.R., and Cubillos-Ruiz, J.R. (2016). Stateof-the-art of regulatory dendritic cells in cancer. Pharmacol. Ther. 164, 97-104.

Deicher, A., Andersson, R., Tingstedt, B., Lindell, G., Bauden, M., and Ansari, D. (2018). Targeting dendritic cells in pancreatic ductal adenocarcinoma. Cancer Cell Int. 18, 85

Dudek, A.M., Martin, S., Garg, A.D., and Agostinis, P. (2013). Immature, semi-mature, and fully mature dendritic cells: toward a DC-cancer cells interface that augments anticancer immunity. Front. Immunol. 4, 438.

Foley, K., Kim, V., Jaffee, E., and Zheng, L. (2016). Current progress in immunotherapy for pancreatic cancer. Cancer Lett. 381, 244-251.

Fukunaga, A., Miyamoto, M., Cho, Y., Murakami, S., Kawarada, Y., Oshikiri, T., Kato, K., Kurokawa, T., Suzuoki, M., Nakakubo, Y., et al. (2004). Cd8+ tumor-infiltrating lymphocytes together with $\mathrm{Cd} 4+$ tumor-infiltrating lymphocytes and dendritic cells improve the prognosis of patients with pancreatic adenocarcinoma. Pancreas 28, e26-e31.

Gabrilovich, D. (2004). Mechanisms and functional significance of tumourinduced dendritic-cell defects. Nat. Rev. Immunol. 4, 941-952.

Giovanelli, P., Sandoval, T.A., and Cubillos-Ruiz, J.R. (2019). Dendritic cell 
metabolism and function in tumors. Trends Immunol. 40, 699-718

He, S., Fei, M., Wu, Y., Zheng, D., Wan, D., Wang, L., and Li, D. (2011). Distribution and clinical significance of Th17 cells in the tumor microenvironment and peripheral blood of pancreatic cancer patients. Int. J. Mol. Sci. 12, 7424-7437.

Hegde, S., Krisnawan, V.E., Herzog, B.H., Zuo, C., Breden, M.A., Knolhoff, B.L., Hogg, G.D., Tang, J.P., Baer, J.M., Mpoy, C., et al. (2020). Dendritic cell paucity leads to dysfunctional immune surveillance in pancreatic cancer. Cancer Cell 37, 289-307.e9.

Hirooka, S., Yanagimoto, H., Satoi, S., Yamamoto, T., Toyokawa, H., Yamaki, S., Yui, R., Inoue, K., Michiura, T., and Kwon, A.H. (2011). The role of circulating dendritic cells in patients with unresectable pancreatic cancer. Anticancer Res. 31, 3827-3834.

Liu, X., Wang, J., Wang, H., Yin, G., Liu, Y., Lei, X., and Xiang, M. (2015). REG3A accelerates pancreatic cancer cell growth under IL-6-associated inflammatory condition: involvement of a REG3A-JAK2/STAT3 positive feedback loop. Cancer Lett. 362, 45-60.

Liu, X., Zhou, Z., Cheng, Q., Wang, H., Cao, H., Xu, Q., Tuo, Y., Jiang, L., Zou, $Y$., Ren, $H_{\text {., }}$ et al. (2017). Acceleration of pancreatic tumorigenesis under immunosuppressive microenvironment induced by Reg3g overexpression. Cell Death Dis. 8, e3033.

Ma, C., Su, M., Shen, K., Chen, J., Ning, Y., and Qi, C. (2020). Key genes and pathways in tumor-educated dendritic cells by bioinformatical analysis. Microbiol. Immunol. 64, 63-71.

Nefedova, Y., Cheng, P., Gilkes, D., Blaskovich, M., Beg, A.A., Sebti, S.M., and Gabrilovich, D.I. (2005). Activation of dendritic cells via inhibition of Jak2/STAT3 signaling. J. Immunol. 175, 4338-4346.

Padoan, A., Plebani, M., and Basso, D. (2019). Inflammation and pancreatic cancer: focus on metabolism, cytokines, and immunity. Int. J. Mol. Sci. 20, 676.

Ren, B., Cui, M., Yang, G., Wang, H., Feng, M., You, L., and Zhao, Y. (2018). Tumor microenvironment participates in metastasis of pancreatic cancer. Mol. Cancer 17, 108.

Takasawa, S. (2016). Regenerating gene (REG) product and its potential clinical usage. Expert Opin. Ther. Targets 20, 541-550.

Tjomsland, V., Spangeus, A., Sandstrom, P., Borch, K., Messmer, D., and Larsson, M. (2010). Semi mature blood dendritic cells exist in patients with ductal pancreatic adenocarcinoma owing to inflammatory factors released from the tumor. PLoS One 5, e13441.

Wang, J., Zhou, H., Han, Y., Liu, X., Wang, M., Wang, X., Yin, G., Li, X., and Xiang, M. (2014). SOCS3 methylation in synergy with Reg3A overexpression promotes cell growth in pancreatic cancer. J. Mol. Med. (Berl.) 92, 1257-1269.

Yamamoto, T., Yanagimoto, H., Satoi, S., Toyokawa, H., Yamao, J., Kim, S., Terakawa, N., Takahashi, K., and Kwon, A.H. (2012). Circulating myeloid dendritic cells as prognostic factors in patients with pancreatic cancer who have undergone surgical resection. J. Surg. Res. 173, 299-308.

Yan, J., Smyth, M.J., and Teng, M.W.L. (2018). Interleukin (II)-12 and II-23 and their conflicting roles in cancer. Cold Spring Harb. Perspect. Biol. 10, a028530.

Yin, G., Du, J., Cao, H., Liu, X., Xu, Q., and Xiang, M. (2015). Reg3g promotes pancreatic carcinogenesis in a murine model of chronic pancreatitis. Dig. Dis. Sci. 60, 3656-3668.

Zhang, M.Y., Wang, J., and Guo, J. (2019). Role of regenerating isletderived protein 3A in gastrointestinal cancer. Front. Oncol. 9, 1449. 